\title{
Edge metric dimension of some classes of circulant graphs
}

\author{
Muhammad Ahsan, Zohaib Zahid, Sohail Zafar.
}

\begin{abstract}
Let $G=(V(G), E(G))$ be a connected graph and $x, y \in V(G)$, $d(x, y)=\min \{$ length of $x-y$ path $\}$ and for $e \in E(G), d(x, e)=$ $\min \{d(x, a), d(x, b)\}$, where $e=a b$. A vertex $x$ distinguishes two edges $e_{1}$ and $e_{2}$, if $d\left(e_{1}, x\right) \neq d\left(e_{2}, x\right)$. Let $W_{E}=\left\{w_{1}, w_{2}, \ldots, w_{k}\right\}$ be an ordered set in $V(G)$ and let $e \in E(G)$. The representation $r\left(e \mid W_{E}\right)$ of $e$ with respect to $W_{E}$ is the $k$-tuple $\left(d\left(e, w_{1}\right), d\left(e, w_{2}\right), \ldots, d\left(e, w_{k}\right)\right)$. If distinct edges of $G$ have distinct representation with respect to $W_{E}$, then $W_{E}$ is called an edge metric generator for $G$. An edge metric generator of minimum cardinality is an edge metric basis for $G$, and its cardinality is called edge metric dimension of $G$, denoted by $\operatorname{edim}(G)$. The circulant graph $C_{n}(1, m)$ has vertex set $\left\{v_{1}, v_{2}, \ldots, v_{n}\right\}$ and edge set $\left\{v_{i} v_{i+1}: 1 \leq\right.$ $i \leq n-1\} \cup\left\{v_{n} v_{1}\right\} \cup\left\{v_{i} v_{i+m}: 1 \leq i \leq n-m\right\} \cup\left\{v_{n-m+i} v_{i}: 1 \leq i \leq m\right\}$. In this paper, it is shown that the edge metric dimension of circulant graphs $C_{n}(1,2)$ and $C_{n}(1,3)$ is constant.
\end{abstract}

\section{Introduction and Preliminaries}

Suppose $G$ is a connected and undirected graph with vertex set $V(G)$ and edge set $E(G)$. The degree $d_{v}$ of a vertex $v \in V(G)$ is the total number of vertices joining to $v$. The maximum and minimum degree of the graph $G$ are represented by $\Delta(G)$ and $\delta(G)$ respectively. Slater introduced the concept of metric

Key Words: Edge metric dimension, edge metric generator, basis, resolving set, circulant graphs

2010 Mathematics Subject Classification: Primary 05C12; Secondary

Received: 9.10 .2019

Accepted: 20.02.2020 
dimension in order to locate an intruder's place in a network [18]. Melter and Harary further discussed the Slater's concept in [4]. The metric dimension, is defined as follows: Let $W=\left\{v_{1}, v_{2}, \ldots, v_{k}\right\}$ be an ordered set of vertices of $G$ and let $v$ be a vertex of $G$. The representation $r(v \mid W)$ of $v$ with respect to $W$ is the $k$-tuple $\left(d\left(v, v_{1}\right), d\left(v, v_{2}\right), \ldots, d\left(v, v_{k}\right)\right)$. If distinct vertices of $G$ have distinct representation with respect to $W$, then $W$ is called resolving set for $G$. A resolving set of minimum cardinality is a metric basis for $G$, and its cardinality is called the metric dimension of $G$, denoted by $\operatorname{dim}(G)$. For more detail on metric dimension, we refer to $[5,9,20]$.

Metric dimension is an essential tool in image processing and pattern recognition (see [14]). In games such as mastermind and coin weighing, the use of metric dimension of graphs was also studied in [1]. In pharmaceutical chemistry, metric dimension of graphs provides distinct representation of different chemical compounds (see $[2,10]$ ). The vertices and edges represent the atoms and bond types of different chemicals respectively. In this way, we can show the structure of chemical compounds by these labeled graphs. For applications in robot navigation, combinatorial optimization and coast guard loran, we refer articles $[17,18,19]$.

Kelenc in [11] extended the idea of metric dimension to edge metric dimension. Let $d(x, e)$ denotes the distance between edge $e$ and vertex $x$, defined as $d(x, e)=\min \{d(x, a), d(x, b)\}$, where $e=a b$ (see [11]). A vertex $x$ distinguishes two edges $e_{1}$ and $e_{2}$, if $d\left(e_{1}, x\right) \neq d\left(e_{2}, x\right)$. Let $W_{E}=\left\{w_{1}, w_{2}, \ldots, w_{k}\right\}$ be an ordered set in $V(G)$ and let $e \in E(G)$. The representation $r\left(e \mid W_{E}\right)$ of $e$ with respect to $W_{E}$ is the $k$-tuple $\left(d\left(e, w_{1}\right), d\left(e, w_{2}\right), \ldots, d\left(e, w_{k}\right)\right)$. If distinct edges of $G$ have distinct representation with respect to $W_{E}$, then $W_{E}$ is called an edge metric generator for $G$ (see [11]). An edge metric generator of minimum cardinality is an edge metric basis for $G$, and its cardinality is called edge metric dimension of $G$, denoted by $\operatorname{edim}(G)$. Kelenc in [11] also computed the edge metric dimension for path, cycle, wheel and complete graphs. In [21], Zubrilina classified the graphs on $n$ vertices for which edge metric dimension is $n-1$. In [13], Kratica computed the edge metric dimension of generalized Petersen graphs $G P(n, k)$ for $k=1,2$ while for the other values of $k$ the lower bound is given. In 2018, Mufti et al. computed the edge metric dimension of barcycentric subdivision of Cayley graphs (see [15]). The following lemmas are helpful for calculating the edge metric dimension of graphs:

Lemma 1.1. [11] edim $(G)=1$ if and only if $G$ is the path graph.

Lemma 1.2. [11] For a connected graph $G$, edim $(G) \geq \log _{2}(\triangle(G))$.

Lemma 1.3. [11] For a connected graph $G$, edim $(G) \geq 1+\left\lceil\log _{2} \delta(G)\right\rceil$.

The circulant graph $C_{n}(1, m)$ has $V\left(C_{n}(1, m)\right)=\left\{v_{1}, v_{2}, \ldots, v_{n}\right\}$ and $E\left(C_{n}(1, m)\right)=\left\{v_{i} v_{i+1}: 1 \leq i \leq n-1\right\} \cup\left\{v_{n} v_{1}\right\} \cup\left\{v_{i} v_{i+m}: 1 \leq i \leq\right.$ 
$n-m\} \cup\left\{v_{n-m+i} v_{i}: 1 \leq i \leq m\right\}$. Circulant graphs are an important class of graphs used in computer sciences especially in designing of computer network topologies and local area networks.

The rest of paper is structured as follows:

In the second section, we will compute the edge metric dimension of the family of circulant graphs $C_{n}(1,2)$. In third section, we will calculate the edge metric dimension of the family of circulant graphs $C_{n}(1,3)$. In last section, we concluded the paper by open problem.

\section{Edge metric dimension of family of circulant graphs $C_{n}(1,2)$}

In this section, we will calculate the edge metric dimension of family of circulant graphs $C_{n}(1,2)$. Figure 1 shows the circulant graph $C_{8}(1,2)$.

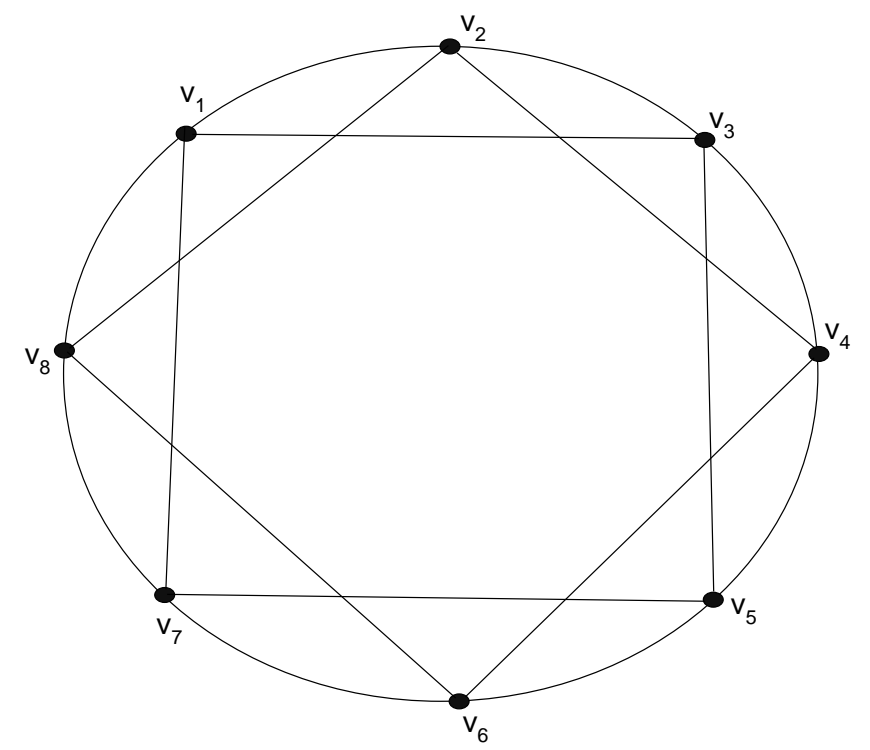

Figure 1: Circulant graph $C_{8}(1,2)$

The following theorem tells us the metric dimension of $C_{n}(1,2)$.

Theorem 2.1. [7, 16] Let $C_{n}(1,2)$ be a circulant graph with $n \geq 5$, then 


$$
\operatorname{dim}\left(C_{n}(1,2)\right)= \begin{cases}3, & \text { if } n \equiv 0,2,3(\bmod 4) \\ \leq 4, & \text { otherwise. }\end{cases}
$$

Now, we will compute the edge metric dimension of $C_{n}(1,2)$.

Theorem 2.2. Let $C_{n}(1,2)$ be a circulant graph with $n \geq 5$, then

$$
\operatorname{edim}\left(C_{n}(1,2)\right)= \begin{cases}5, & \text { if } n \equiv 1,2(\bmod 4) \\ 4, & \text { otherwise }\end{cases}
$$

Proof. In order to compute edge metric dimension of $C_{n}(1,2)$, we have the following cases.

Case $(\mathbf{i})$ : When $n \equiv 0(\bmod 4)$ and $n \geq 5$.

Let $n=4 k, k \geq 2, k \in \mathbf{Z}^{+}$and $W_{E}=\left\{v_{1}, v_{2}, v_{2 k}, v_{2 k+1}\right\} \subset V\left(C_{n}(1,2)\right)$, we have to show that $W_{E}$ is an edge metric generator of $C_{n}(1,2)$. For this, we give representations of each edge of $C_{n}(1,2)$.

$$
\begin{aligned}
& r\left(v_{2 i+1} v_{2 i+2} \mid W_{E}\right)= \begin{cases}(i, i, k-i-1, k-i), & \text { if } 0 \leq i \leq k-1 ; \\
(k, k, 1,0), & \text { if } i=k ; \\
(2 k-i, 2 k-i, i-k+1, i-k), & \text { if } k+1 \leq i \leq 2 k-1 ;\end{cases} \\
& r\left(v_{2 i+1} v_{2 i+3} \mid W_{E}\right)= \begin{cases}(0,1, k-1, k-1), & \text { if } i=0 ; \\
(i, i, k-i-1, k-i-1), & \text { if } 1 \leq i \leq k-2 ; \\
(k-1, k-1,1,0), & \text { if } i=k-1 ; \\
(2 k-i-1,2 k-i, i-k+1, i-k), & \text { if } k \leq i \leq 2 k-2 ;\end{cases} \\
& r\left(v_{n-1} v_{1} \mid W_{E}\right)=(0,1, k, k-1), \\
& r\left(v_{2 i} v_{2 i+1} \mid W_{E}\right)= \begin{cases}(i, i-1, k-i, k-i), & \text { if } 1 \leq i \leq k ; \\
(2 k-i, 2 k-i+1, i-k, i-k), & \text { if } k+1 \leq i \leq 2 k-1 ;\end{cases} \\
& r\left(v_{n} v_{1} \mid W_{E}\right)=(0,1, k, k), \quad \text { if } 1 \leq i \leq k-1 ; \\
& r\left(v_{2 i} v_{2 i+2} \mid W_{E}\right)= \begin{cases}(i, i-1, k-i-1, k-i), & \text { if } i=k ; \\
(k, k-1,0,1), & \text { if } k+1 \leq i \leq 2 k-1 ;\end{cases}
\end{aligned}
$$


and $r\left(v_{n} v_{2} \mid W_{E}\right)=(1,0, k-1, k)$.

We see that there are no two edges having the same representations. This shows that $\operatorname{edim}\left(C_{n}(1,2)\right) \leq 4$.

On the other hand, we have to show that edim $\left(C_{n}(1,2)\right) \geq 4$. For this purpose, we have to show that there is no edge metric generator have cardinality 3 , we suppose on contrary that $\operatorname{edim}\left(C_{n}(1,2)\right)=3$ and let $W_{E}=\left\{v_{1}, v_{i}, v_{j}\right\}$. Then the Table 1 shows all order pairs of edges $(e, f)$ for which $r\left(e \mid W_{E}\right)=r\left(f \mid W_{E}\right)$.

\begin{tabular}{|c|c|}
\hline Conditions on $i$ and $j$ & $(e, f)$ \\
\hline $2 \leq i \leq j \leq 2 k$ & $\left(v_{n} v_{1}, v_{n-1} v_{1}\right)$ \\
\hline $2 \leq i \leq 2 k, 2 k+1 \leq j \leq n-1$ and $j$ is even & $\left(v_{n} v_{1}, v_{n-1} v_{1}\right)$ \\
\hline $2 \leq i \leq 2 k-1$ and $j=n$ & $\left(v_{n-1} v_{n}, v_{n-2} v_{n}\right)$ \\
\hline$i=2 k$ and $j=n$ & $\left(v_{1} v_{2}, v_{1} v_{3}\right)$ \\
\hline $2 \leq i \leq 2 k-1,2 k+1 \leq j \leq n-2$ and $j$ is odd & $\left(v_{n-1} v_{n}, v_{n-2} v_{n}\right)$ \\
\hline$i=2 k, 2 k+2 \leq j \leq n$ and $j$ is odd & $\left(v_{1} v_{2}, v_{1} v_{3}\right)$ \\
\hline$i=2 k$ and $j=2 k+1$ & $\left(v_{2 k-1} v_{2 k}, v_{2 k-2} v_{2 k}\right)$ \\
\hline $2 \leq i \leq 2 k-1$ and $j=n-1$ & $\left(v_{2 k} v_{2 k+1}, v_{2 k} v_{2 k+2}\right)$ \\
\hline
\end{tabular}

Table 1: $(e, f)$ for which $r\left(e \mid W_{E}\right)=r\left(f \mid W_{E}\right)$

In all possibilities, we conclude that there is no edge metric generator of 3 vertices. Hence edim $\left(C_{n}(1,2)\right)=4$.

Case (ii): When $n \equiv 1(\bmod 4)$ and $n \geq 5$.

Let $n=4 k+1, k \geq 1, k \in \mathbf{Z}^{+}$and $W_{E}=\left\{v_{1}, v_{2}, v_{3}, v_{2 k+2}, v_{2 k+3}\right\} \subset$ $V\left(C_{n}(1,2)\right)$, we have to show that $W_{E}$ is an edge metric generator of $C_{n}(1,2)$. For this, we give representations of each edge of $C_{n}(1,2)$.

$r\left(v_{2 i+1} v_{2 i+2} \mid W_{E}\right)= \begin{cases}(0,0,1, k, k), & \text { if } i=0 ; \\ (i, i, i-1, k-i, k-i+1), & \text { if } 1 \leq i \leq k ; \\ (2 k-i, 2 k-i+1,2 k-i+1, & \\ i-k, i-k-1), & \text { if } k+1 \leq i \leq 2 k-1 ;\end{cases}$

$r\left(v_{n} v_{1} \mid W_{E}\right)=(0,1,1, k, k-1)$,

$r\left(v_{2 i+1} v_{2 i+3} \mid W_{E}\right)= \begin{cases}(0,1,0, k, k), & \text { if } i=0 ; \\ (i, i, i-1, k-i, k-i), & \text { if } 1 \leq i \leq k-1 ; \\ (k, k, k-1,1,0), & \text { if } i=k ; \\ (2 k-i, 2 k-i, 2 k-i+1, & \\ i-k, i-k-1), & \text { if } k+1 \leq i \leq 2 k-1 ;\end{cases}$

$r\left(v_{n} v_{2} \mid W_{E}\right)=(1,0,1, k, k-1)$, 


$$
\begin{aligned}
& r\left(v_{2 i} v_{2 i+1} \mid W_{E}\right)= \begin{cases}(i, i-1, i-1, k-i, k-i), & \text { if } 1 \leq i \leq k ; \\
(k, k, k, 0,0), & \text { if } i=k+1 ; \\
(2 k-i+1,2 k-i+1,2 k-i+2, & \text { if } k+2 \leq i \leq 2 k ;\end{cases} \\
& r\left(v_{n-1} v_{1} \mid W_{E}\right)=(0,1,1, k-1, k-1) \text { and } \\
& r\left(v_{2 i} v_{2 i+2} \mid W_{E}\right)= \begin{cases}(1,0,1, k-1, k), & \text { if } i=1 ; \\
(i, i-1, i-1, k-i, k-i+1), & \text { if } 2 \leq i \leq k ; \\
(k-1, k, k, 0,1), & \text { if } i=k+1 ; \\
(2 k-i, 2 k-i+1,2 k-i+1, & \text { if } k+2 \leq i \leq 2 k-1 . i-k-1),\end{cases}
\end{aligned}
$$

We see that there are no two edges having the same representations. This shows that $\operatorname{edim}\left(C_{n}(1,2)\right) \leq 5$.

On the other hand, we have to show that $\operatorname{edim}\left(C_{n}(1,2)\right) \geq 5$. For this purpose, we have to show that there is no edge metric generator have cardinality 4 , we suppose on contrary that $\operatorname{edim}\left(C_{n}(1,2)\right)=4$ and let $W_{E}=\left\{v_{1}, v_{i}, v_{j}, v_{l}\right\}$. Then the Table 2 shows all order pairs of edges $(e, f)$ for which $r\left(e \mid W_{E}\right)=$ $r\left(f \mid W_{E}\right)$.

\begin{tabular}{|c|c|}
\hline Conditions on $i, j$ and $l$ & $(e, f)$ \\
\hline $2 \leq i \leq j \leq l \leq 2 k$ & $\left(v_{n} v_{1}, v_{n-1} v_{1}\right)$ \\
\hline $2 \leq i \leq j \leq 2 k, 2 k+1 \leq l \leq n-2$ and $l$ is even & $\left(v_{n-1} v_{n}, v_{n-2} v_{n}\right)$ \\
\hline $2 \leq i \leq j \leq 2 k$ and $l=n-1$ & $\left(v_{2 k+1} v_{2 k+2}, v_{2 k+1} v_{2 k+3}\right)$ \\
\hline $2 \leq i \leq j \leq 2 k, 2 k+1 \leq l \leq n-1$ and $l$ is odd & $\left(v_{n} v_{1}, v_{n-1} v_{1}\right)$ \\
\hline $2 \leq i \leq j \leq 2 k$ and $l=n$ & $\left(v_{n} v_{n-1}, v_{n} v_{n-2}\right)$ \\
\hline
\end{tabular}

Table 2: $(e, f)$ for which $r\left(e \mid W_{E}\right)=r\left(f \mid W_{E}\right)$

Since in each case we get contradiction. We conclude that there is no edge metric generator of 4 vertices. So it is obvious that edge metric generator of 3 vertices does not exist. Hence $\operatorname{edim}\left(C_{n}(1,2)\right)=5$.

Case (iii): When $n \equiv 2(\bmod 4)$ and $n \geq 5$.

Let $n=4 k+2, k \geq 2, k \in \mathbf{Z}^{+}$and $W_{E}=\left\{v_{1}, v_{2}, v_{3}, v_{2 k+2}, v_{2 k+3}\right\} \quad \subset$ $V\left(C_{n}(1,2)\right)$, we have to show that $W_{E}$ is an edge metric generator of $C_{n}(1,2)$. For this, we give representations of each edge of $C_{n}(1,2)$. 


$$
\begin{aligned}
& r\left(v_{2 i+1} v_{2 i+2} \mid W_{E}\right)= \begin{cases}(0,0,1, k, k), & \text { if } i=0 ; \\
(i, i, i-1, k-i, k+1-i), & \text { if } 1 \leq i \leq k ; \\
(k, k, k, 1,2), & \text { if } i=k+1 ; \\
(2 k-i, 2 k-i, 2 k-i+1, & \\
i-k, i-k+1), & \text { if } k+2 \leq i \leq 2 k ;\end{cases} \\
& r\left(v_{2 i+1} v_{2 i+3} \mid W_{E}\right)= \begin{cases}(0,1,0, k, k), & \text { if } i=0 ; \\
(i, i, i-1, k-i, k-i), & \text { if } 1 \leq i \leq k-1 ; \\
(k, k, k-1,1,0), & \text { if } i=k ; \\
(2 k-i, 2 k-i+1,2 k-i+1, & \\
i-k, i-k-1), & \text { if } k+1 \leq i \leq 2 k-1 ;\end{cases} \\
& r\left(v_{n-1} v_{1} \mid W_{E}\right)=(0,1,1, k, k-1) \text {, } \\
& r\left(v_{2 i} v_{2 i+1} \mid W_{E}\right)= \begin{cases}(i, i-1, i-1, k+1-i, k+1-i), & \text { if } 1 \leq i \leq k ; \\
(k, k, k, 0,0), & \text { if } i=k+1 ; \\
(2 k-i+1,2 k-i+2,2 k-i+2, & \\
i-k-1, i-k-1), & \text { if } k+2 \leq i \leq 2 k ;\end{cases} \\
& r\left(v_{n} v_{1} \mid W_{E}\right)=(0,1,1, k, k), \\
& r\left(v_{2 i} v_{2 i+2} \mid W_{E}\right)= \begin{cases}(1,0,1, k-1, k), & \text { if } i=1 ; \\
(i, i-1, i-1, k-i, k+1-i), & \text { if } 2 \leq i \leq k ; \\
(k, k, k, 0,1), & \text { if } i=k+1 ; \\
(2 k+1-i, 2 k+1-i, 2 k-i+2, & \\
i-k-1, i-k-1), & \text { if } k+2 \leq i \leq 2 k ;\end{cases}
\end{aligned}
$$

and $r\left(v_{n} v_{2} \mid W_{E}\right)=(1,0,1, k, k)$.

We see that there are no two edges having the same representations. This shows that $\operatorname{edim}\left(C_{n}(1,2)\right) \leq 5$.

On the other hand, we have to show that $\operatorname{edim}\left(C_{n}(1,2)\right) \geq 5$. For this purpose, we have to show that there is no edge metric generator have cardinality 4 , we suppose on contrary that $\operatorname{edim}\left(C_{n}(1,2)\right)=4$ and let $W_{E}=\left\{v_{1}, v_{i}, v_{j}, v_{l}\right\}$. Then the Table 3 shows all order pairs of edges $(e, f)$ for which $r\left(e \mid W_{E}\right)=$ $r\left(f \mid W_{E}\right)$. 


\begin{tabular}{|c|c|}
\hline Conditions on $i, j$ and $l$ & $(e, f)$ \\
\hline $2 \leq i \leq j \leq l \leq 2 k+1$ & $\left(v_{n} v_{1}, v_{n-1} v_{1}\right)$ \\
\hline $2 \leq i \leq j \leq 2 k+1,2 k+2 \leq l \leq n-1$ and \\
$l$ is even & $\left(v_{n} v_{1}, v_{n-1} v_{1}\right)$ \\
\hline $2 \leq i \leq j \leq 2 k+1$ and $l=n$ & $\left(v_{2 k+1} v_{2 k+2}, v_{2 k+1} v_{2 k+3}\right)$ \\
\hline $2 \leq i \leq j \leq 2 k+1,2 k+2 \leq l \leq n-2$ and \\
$l$ is odd & $\left(v_{n} v_{n-1}, v_{n} v_{n-2}\right)$ \\
\hline $2 \leq i \leq j \leq 2 k+1$ and $l=n-1$ & $\left(v_{2 k+2} v_{2 k+3}, v_{2 k+2} v_{2 k+4}\right)$ \\
\hline
\end{tabular}

Table 3: $(e, f)$ for which $r\left(e \mid W_{E}\right)=r\left(f \mid W_{E}\right)$

Since in each case we get contradiction. We conclude that there is no edge metric generator of 4 vertices. So it is obvious that edge metric generator of 3 vertices does not exist. Hence $\operatorname{edim}\left(C_{n}(1,2)\right)=5$.

Case (iv): When $n \equiv 3(\bmod 4)$ and $n \geq 5$.

Let $n=4 k+3, k \geq 2, k \in \mathbf{Z}^{+}$and $W_{E}=\left\{v_{1}, v_{2}, v_{2 k+1}, v_{2 k+2}\right\} \subset V\left(C_{n}(1,2)\right)$, we have to show that $W_{E}$ is an edge metric generator of $C_{n}(1,2)$. For this, we give representations of each edge of $C_{n}(1,2)$.

$r\left(v_{2 i+1} v_{2 i+2} \mid W_{E}\right)= \begin{cases}(i, i, k-i, k-i), & \text { if } 0 \leq i \leq k ; \\ (2 k+1-i, 2 k-i+2, i-k, i-k), & \text { if } k+1 \leq i \leq 2 k ;\end{cases}$ $r\left(v_{n} v_{1} \mid W_{E}\right)=(0,1, k, k+1)$,

$r\left(v_{2 i+1} v_{2 i+3} \mid W_{E}\right)= \begin{cases}(0,1, k-1, k), & \text { if } i=0 ; \\ (i, i, k-i-1, k-i), & \text { if } 1 \leq i \leq k-1 ; \\ (k, k, 0,1), & \text { if } i=k ; \\ (2 k+1-i, 2 k+1-i, i-k, i-k), & \text { if } k+1 \leq i \leq 2 k ;\end{cases}$

$r\left(v_{n} v_{2} \mid W_{E}\right)=(1,0, k, k)$,

$r\left(v_{2 i} v_{2 i+1} \mid W_{E}\right)= \begin{cases}(i, i-1, k-i, k+1-i), & \text { if } 1 \leq i \leq k ; \\ (k+1, k, 1,0), & \text { if } i=k+1 ; \\ (2 k-i+2,2 k-i+2, i-k, & \\ i-k-1), & \text { if } k+2 \leq i \leq 2 k+1 ;\end{cases}$

$r\left(v_{n-1} v_{1} \mid W_{E}\right)=(0,1, k, k)$ and 


$$
r\left(v_{2 i} v_{2 i+2} \mid W_{E}\right)= \begin{cases}(i, i-1, k-i, k-i), & \text { if } 1 \leq i \leq k-1 ; \\ (k, k-1,1,0), & \text { if } i=k ; \\ (k, k, 1,0), & \text { if } i=k+1 ; \\ (2 k+1-i, 2 k-i+2, i-k, & \\ i-k-1), & \text { if } k+2 \leq i \leq 2 k .\end{cases}
$$

We see that there are no two edges having the same representations. This shows that $\operatorname{edim}\left(C_{n}(1,2)\right) \leq 4$.

On the other hand, we have to show that $\operatorname{edim}\left(C_{n}(1,2)\right) \geq 4$. For this purpose, we have to show that there is no edge metric generator have cardinality 3 , we suppose on contrary that $\operatorname{edim}\left(C_{n}(1,2)\right)=3$ and let $W_{E}=\left\{v_{1}, v_{i}, v_{j}\right\}$. Then the Table 4 shows all order pairs of edges $(e, f)$ for which $r\left(e \mid W_{E}\right)=r\left(f \mid W_{E}\right)$.

\begin{tabular}{|c|c|}
\hline Conditions on $i$ and $j$ & $(e, f)$ \\
\hline $2 \leq i \leq j \leq 2 k+1$ & $\left(v_{n} v_{1}, v_{n-1} v_{1}\right)$ \\
\hline $2 \leq i \leq 2 k, 2 k+2 \leq j \leq n-2$ and $j$ is even & $\left(v_{n} v_{n-1}, v_{n} v_{n-2}\right)$ \\
\hline$i=2 k+1,2 k+3 \leq j \leq n$ and $j$ is even & $\left(v_{2 k+1} v_{2 k+2}, v_{2 k+1} v_{2 k+3}\right)$ \\
\hline$i=2 k+1$ and $j=2 k+2$ & $\left(v_{n} v_{2}, v_{n} v_{n-2}\right)$ \\
\hline $2 \leq i \leq 2 k+1$ and $j=n-1$ & $\left(v_{2 k+1} v_{2 k+2}, v_{2 k+1} v_{2 k+3}\right)$ \\
\hline $2 \leq i \leq 2 k+1,2 k+2 \leq j \leq n-1$ and $j$ is odd & $\left(v_{n} v_{1}, v_{n-1} v_{1}\right)$ \\
\hline $2 \leq i \leq 2 k+1, j=n$ and $i \neq 2 k$ & $\left(v_{2 k} v_{2 k+2}, v_{2 k+2} v_{2 k+4}\right)$ \\
\hline$i=2 k$ and $j=n$ & $\left(v_{2 k} v_{2 k+1}, v_{2 k} v_{2 k+2}\right)$ \\
\hline
\end{tabular}

Table 4: $(e, f)$ for which $r\left(e \mid W_{E}\right)=r\left(f \mid W_{E}\right)$

In all possibilities, we conclude that there is no edge metric generator of 3 vertices. Hence $\operatorname{edim}\left(C_{n}(1,2)\right)=4$.

\section{Edge metric dimension of family of circulant graphs $C_{n}(1,3)$}

In this section, we will compute the edge metric dimension of family of circulant graphs $C_{n}(1,3)$. Figure 2 shows the circulant graph $C_{16}(1,3)$.

The following theorem tells us the metric dimension of $C_{n}(1,3)$.

Theorem 3.1. $[6,8,16]$ Let $C_{n}(1,3)$ be a circulant graph with $n \geq 6$, then 


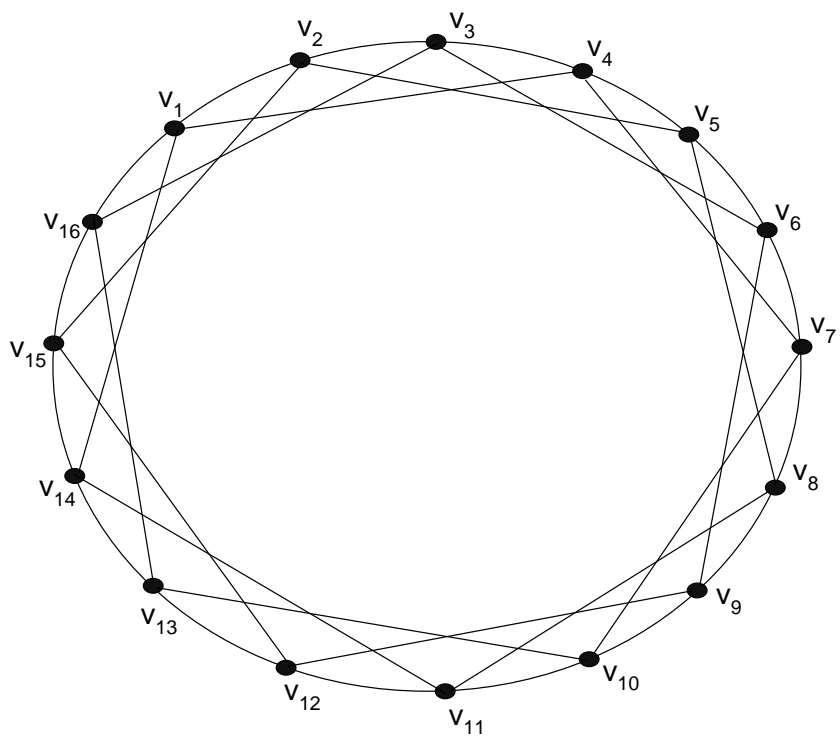

Figure 2: Circulant graph $C_{16}(1,3)$

$$
\operatorname{dim}\left(C_{n}(1,3)\right)= \begin{cases}3, & \text { if } n \equiv 1(\bmod 6) \\ 4, & \text { if } n \equiv 0,3,4,5(\bmod 6) \\ 4 \leq \operatorname{dim}\left(C_{n}(1,3)\right) \leq 6, & \text { if } n \equiv 2(\bmod 6)\end{cases}
$$

Now, we will compute the edge metric dimension of $C_{n}(1,3)$.

Theorem 3.2. Let $C_{n}(1,3)$ be a circulant graph with $n \geq 6$, then

$$
\operatorname{edim}\left(C_{n}(1,3)\right)= \begin{cases}6, & n=8 \\ 5, & \text { if } n \equiv 2,5(\bmod 6) \text { and } n \neq 8 \\ 4, & \text { otherwise }\end{cases}
$$


Proof. In order to compute edge metric dimension of $C_{n}(1,3)$, we have the following cases.

Case (i): When $n=7, W_{E}=\left\{v_{1}, v_{2}, v_{4}, v_{5}\right\} \subset V\left(C_{7}(1,3)\right)$ is edge metric generator and hence $\operatorname{edim}\left(C_{7}(1,3)\right)=4$.

Case (ii): When $n=8, W_{E}=\left\{v_{1}, v_{2}, v_{3}, v_{4}, v_{5}, v_{6}\right\} \subset V\left(C_{8}(1,3)\right)$ is edge metric generator and hence $\operatorname{edim}\left(C_{8}(1,3)\right)=6$.

Case (iii): When $n \equiv 0(\bmod 6)$ and $n \geq 6$.

Let $n=6 k, k \geq 1, k \in \mathbf{Z}^{+}$and $W_{E}=\left\{v_{1}, v_{2}, v_{3}, v_{4}\right\} \subset V\left(C_{n}(1,3)\right)$, we have to show that $W_{E}$ is an edge metric generator of $C_{n}(1,3)$. For this, we give representations of each edge of $C_{n}(1,3)$.

$$
\begin{aligned}
& r\left(v_{3 i+1} v_{3 i+2} \mid W_{E}\right)= \begin{cases}(0,0,1,1), & \text { if } i=0 ; \\
(i, i, i, i-1), & \text { if } 1 \leq i \leq k ; \\
(2 k-i, 2 k-i, 2 k-i+1, & \\
2 k-i+1), & \text { if } k+1 \leq i \leq 2 k-1\end{cases} \\
& r\left(v_{3 i+2} v_{3 i+3} \mid W_{E}\right)= \begin{cases}(1,0,0,1), & \text { if } i=0 ; \\
(i+1, i, i, i), & \text { if } 1 \leq i \leq k-1 ; \\
(k, k, k, k), & \text { if } i=k ; \\
(2 k-i, 2 k-i, 2 k-i, 2 k-i+1), & \text { if } k+1 \leq i \leq 2 k ;\end{cases} \\
& r\left(v_{3 i} v_{3 i+1} \mid W_{E}\right)= \begin{cases}(i, i, i-1, i-1), & \text { if } 1 \leq i \leq k ; \\
(2 k-i, 2 k-i+1,2 k-i+1, & \\
2 k-i+1), & \text { if } k+1 \leq i \leq 2 k-1 ;\end{cases} \\
& r\left(v_{n} v_{1} \mid W_{E}\right)=(0,1,1,1) \text {, } \\
& r\left(v_{3 i+1} v_{3 i+4} \mid W_{E}\right)= \begin{cases}(0,1,1,0), & \text { if } i=0 ; \\
(i, i+1, i, i-1), & \text { if } 1 \leq i \leq k-1 ; \\
(k-1, k, k, k-1), & \text { if } i=k ; \\
(2 k-i-1,2 k-i, 2 k-i+1, & \\
2 k-i), & \text { if } k+1 \leq i \leq 2 k-1 ;\end{cases} \\
& r\left(v_{n-2} v_{1} \mid W_{E}\right)=(0,1,2,1),
\end{aligned}
$$


$r\left(v_{3 i+2} v_{3 i+5} \mid W_{E}\right)= \begin{cases}(1,0,1,1), & \text { if } i=0 ; \\ (i+1, i, i+1, i), & \text { if } 1 \leq i \leq k-1 ; \\ (k, k-1, k, k), & \text { if } i=k ; \\ (2 k-i, 2 k-i-1,2 k-i, & \\ 2 k-i+1), & \text { if } k+1 \leq i \leq 2 k-2 ;\end{cases}$

$r\left(v_{n-1} v_{2} \mid W_{E}\right)=(1,2,1,2)$,

$r\left(v_{3 i} v_{3 i+3} \mid W_{E}\right)= \begin{cases}(i+1, i, i-1, i), & \text { if } 1 \leq i \leq k-1 ; \\ (k, k, k-1, k), & \text { if } i=k ; \\ (2 k-i, 2 k-i+1,2 k-i, & \\ 2 k-i+1), & \text { if } k+1 \leq i \leq 2 k-1 ;\end{cases}$

and $r\left(v_{n} v_{3} \mid W_{E}\right)=(1,1,0,1)$.

We see that there are no two edges having the same representations. This shows that $\operatorname{edim}\left(C_{n}(1,3)\right) \leq 4$.

On the other hand, we have to show that $\operatorname{edim}\left(C_{n}(1,3)\right) \geq 4$. For this purpose, we have to show that there is no edge metric generator have cardinality 3 , we suppose on contrary that $\operatorname{edim}\left(C_{n}(1,3)\right)=3$ and let $W_{E}=\left\{v_{1}, v_{i}, v_{j}\right\}$. Then the Table 5 shows all order pairs of edges $(e, f)$ for which $r\left(e \mid W_{E}\right)=r\left(f \mid W_{E}\right)$.

\begin{tabular}{|c|c|}
\hline Conditions on $i$ and $j$ & $(e, f)$ \\
\hline $2 \leq i \leq j \leq \frac{n}{2}$ & $\left(v_{n} v_{1}, v_{n-2} v_{1}\right)$ \\
\hline $2 \leq i \leq \frac{n}{2}, \frac{n}{2}+1 \leq j \leq n$ and & \\
$j \neq\left\{\frac{n}{2}+1, \frac{n}{2}+4, \frac{n}{2}+7, \ldots, n-2, n\right\}$ & $\left(v_{n} v_{1}, v_{n-2} v_{1}\right)$ \\
\hline $2 \leq i \leq \frac{n}{2}-1, \frac{n}{2}+1 \leq j \leq n$ and & \\
$j \neq\left\{\frac{n}{2}+3, \frac{n}{2}+6, \frac{n}{2}+9, \ldots, n-3, n-1\right\}$ & $\left(v_{n-1} v_{n}, v_{n-3} v_{n}\right)$ \\
\hline$i=\frac{n}{2}, \frac{n}{2}+1 \leq j \leq n$ and & \\
$j \neq\left\{\frac{n}{2}+3, \frac{n}{2}+6, \frac{n}{2}+9, \ldots, n-3, n-1\right\}$ & $\left(v_{1} v_{2}, v_{1} v_{4}\right)$ \\
\hline
\end{tabular}

Table 5: $(e, f)$ for which $r\left(e \mid W_{E}\right)=r\left(f \mid W_{E}\right)$

In all possibilities, we get contradiction so it concludes that there is no edge metric generator of 3 vertices. Hence $\operatorname{edim}\left(C_{n}(1,3)\right)=4$, for $n \equiv 0(\bmod 6)$. Case (iv): When $n \equiv 1(\bmod 6)$ and $n \geq 13$.

Let $n=6 k+1, k \geq 2, k \in \mathbf{Z}^{+}$and $W_{E}=\left\{v_{1}, v_{3}, v_{5}, v_{n-1}\right\} \subset V\left(C_{n}(1,3)\right)$, we have to show that $W_{E}$ is an edge metric generator of $C_{n}(1,3)$. For this, following are the representations of each edge of $C_{n}(1,3)$. 


$$
\begin{aligned}
& r\left(v_{3 i+1} v_{3 i+2} \mid W_{E}\right)=\left\{\begin{array}{ll}
(0,1,1,1), & \text { if } i=0 ; \\
(i, i, i-1, i+1), & \text { if } 1 \leq i \leq k-1 ; \\
(k, k, k-1, k), & \text { if } i=k ; \\
(k-1, k, k, k-1), & \text { if } i=k+1 ; \\
(2 k-i, 2 k-i+1,2 k-i+2, & \text { if } k+2 \leq i \leq 2 k-1 ; \\
2 k-i), &
\end{array} r\left(v_{n} v_{1} \mid W_{E}\right)=(0,1,2,1),\right.
\end{aligned}
$$$$
r\left(v_{3 i+2} v_{3 i+3} \mid W_{E}\right)= \begin{cases}(1,0,1,1), & \text { if } i=0 ; \\ (i+1, i, i-1, i+1), & \text { if } 1 \leq i \leq k-1 ; \\ (k, k, k-1, k-1), & \text { if } i=k ; \\ (2 k-i, 2 k-i+1,2 k-i+1, & \\ 2 k-i-1), & \text { if } k+1 \leq i \leq 2 k-1 ;\end{cases}
$$$$
r\left(v_{3 i} v_{3 i+1} \mid W_{E}\right)= \begin{cases}(1,0,1,2), & \text { if } i=1 ; \\ (i, i-1, i-1, i+1), & \text { if } 2 \leq i \leq k-1 ; \\ (k, k-1, k-1, k), & \text { if } i=k ; \\ (k, k, k, k-1), & \text { if } i=k+1 ; \\ (2 k-i+1,2 k-i+1,2 k-i+2, & \text { if } k+2 \leq i \leq 2 k ; \\ 2 k-i), & \end{cases}
$$$$
r\left(v_{3 i+1} v_{3 i+4} \mid W_{E}\right)= \begin{cases}(0,1,1,2), & \text { if } i=0 ; \\ (i, i, i, i+2), & \text { if } 1 \leq i \leq k-1 ; \\ (k, k, k, k), & \text { if } i=k ; \\ (2 k-i, 2 k-i, 2 k-i+2, & \\ 2 k-i), & \text { if } k+1 \leq i \leq 2 k-1 ;\end{cases}
$$

$r\left(v_{n} v_{3} \mid W_{E}\right)=(1,0,2,1)$,

$$
r\left(v_{3 i+2} v_{3 i+5} \mid W_{E}\right)= \begin{cases}(1,1,0,1), & \text { if } i=0 ; \\ (i+1, i+1, i-1, i+1), & \text { if } 1 \leq i \leq k-1 ; \\ (2 k-i-1,2 k-i+1,2 k-i-1, & \\ 2 k-i-1), & \text { if } k \leq i \leq 2 k-3 ; \\ (1,3,3,1), & \text { if } i=2 k-2 ;\end{cases}
$$

$r\left(v_{n-2} v_{1} \mid W_{E}\right)=(0,2,2,1)$, 
$r\left(v_{3 i} v_{3 i+3} \mid W_{E}\right)= \begin{cases}(2,0,1,2), & \text { if } i=1 ; \\ (i+1, i-1, i-1, i+1), & \text { if } 2 \leq i \leq k-1 \\ (k+1, k-1, k-1, k-1), & \text { if } i=k ; \\ (2 k-i+1,2 k-i+1,2 k-i+1, & \\ 2 k-i-1), & \text { if } k+1 \leq i \leq 2 k-1\end{cases}$

and $r\left(v_{n-1} v_{2} \mid W_{E}\right)=(1,1,1,0)$.

We see that there are no two edges having the same representations. This shows that $\operatorname{edim}\left(C_{n}(1,3)\right) \leq 4$.

On the other hand, we have to show that $\operatorname{edim}\left(C_{n}(1,3)\right) \geq 4$. For this purpose, we have to show that there is no edge metric generator have cardinality 3 , we suppose on contrary that $\operatorname{edim}\left(C_{n}(1,3)\right)=3$ and let $W_{E}=\left\{v_{1}, v_{i}, v_{j}\right\}$. Then the Table 6 shows all order pairs of edges $(e, f)$ for which $r\left(e \mid W_{E}\right)=r\left(f \mid W_{E}\right)$.

\begin{tabular}{|c|c|}
\hline Conditions on $i, j$ & $(e, f)$ \\
\hline $2 \leq i \leq j \leq \frac{n+1}{2}$ & $\left(v_{n} v_{1}, v_{n-2} v_{1}\right)$ \\
\hline $2 \leq i \leq \frac{n-1}{2}, \frac{n+1}{2} \leq j \leq n$ and & \\
$j \neq\left\{\frac{n+1}{2}+1, \frac{n+1}{2}+4, \frac{n+1}{2}+7, \ldots, n-2, n\right\}$ & $\left(v_{n} v_{1}, v_{n-2} v_{1}\right)$ \\
\hline $2 \leq i \leq \frac{n-1}{2}, \frac{n+1}{2} \leq j \leq n$ and & \\
$j \neq\left\{\frac{n+1}{2}+3, \frac{n+1}{2}+6, \frac{n+1}{2}+9, \ldots, n-3, n-1\right\}$ & $\left(v_{n-1} v_{n}, v_{n-3} v_{n}\right)$ \\
\hline
\end{tabular}

Table 6: $(e, f)$ for which $r\left(e \mid W_{E}\right)=r\left(f \mid W_{E}\right)$

In all possibilities, we get contradiction so it concludes that there is no edge metric generator of 3 vertices. Hence edim $\left(C_{n}(1,3)\right)=4$, for $n \equiv 1(\bmod 6)$.

Case $(\mathbf{v})$ : When $n \equiv 2(\bmod 6)$ and $n \geq 14$.

Let $n=6 k+2, k \geq 2, k \in \mathbf{Z}^{+}$and $W_{E}=\left\{v_{1}, v_{2}, v_{\frac{n}{2}-2}, v_{\frac{n}{2}-1}, v_{n-2}\right\} \quad \subset$ $V\left(C_{n}(1,3)\right)$, we have to show that $W_{E}$ is an edge metric generator of $C_{n}(1,3)$.

For this, we give representations of each edge of $C_{n}(1,3)$.

$r\left(v_{3 i+1} v_{3 i+2} \mid W_{E}\right)= \begin{cases}(i, i, k-i-1, k-i, i+1), & \text { if } 0 \leq i \leq k-1 ; \\ (k, k, 1,1, k), & \text { if } i=k ; \\ (2 k-i+1,2 k-i+1, i-k+1, & \\ i-k+1,2 k-i), & \text { if } k+1 \leq i \leq 2 k-1 ; \\ (1,1, k, k, 1), & \text { if } i=2 k ;\end{cases}$ 


$$
r\left(v_{3 i+2} v_{3 i+3} \mid W_{E}\right)= \begin{cases}(i+1, i, k-i-1, k-i-1, i+2), & \text { if } 0 \leq i \leq k-2 ; \\ (k, k-1,0,0, k), & \text { if } i=k-1 ; \\ (k, k, k+1,1, k-1), & \text { if } i=k ; \\ (2 k-i, 2 k-i+1, i-k+1, & \text { if } k+1 \leq i \leq 2 k-1 ; \\ i-k+1,2 k-i-1), & \end{cases}
$$

$r\left(v_{n} v_{1} \mid W_{E}\right)=(0,1, k, k, 1)$,

$$
\left.\begin{array}{l}
r\left(v_{3 i} v_{3 i+1} \mid W_{E}\right)= \begin{cases}(i, i, k-i, k-i, i+1), & \text { if } 1 \leq i \leq k-1 ; \\
(k, k, 1,0, k), & \text { if } i=k ; \\
(2 k-i+1,2 k-i+1, i-k+1, & \\
(1,1, k, k, 0), & \text { if } k+1 \leq i \leq 2 k-1 ;\end{cases} \\
r\left(v_{3 i+1} v_{3 i+4} \mid W_{E}\right)= \begin{cases}(i, i+1, k-i-1, k-i, i+1), & \text { if } 0 \leq i \leq k-2 ; \\
(k-1, k, 1,1, k), & \text { if } i=2 k ; \\
(k, k, 2,1, k), & \text { if } i=k ;\end{cases} \\
i-k+1,2 k-i), \\
(2,1, k, k, 1),
\end{array}\right)
$$

$r\left(v_{n-1} v_{2} \mid W_{E}\right)=(1,0, k-1, k, 1)$,

$$
r\left(v_{3 i+2} v_{3 i+5} \mid W_{E}\right)= \begin{cases}(i+1, i, k-i-2, k-i-1, i+2), & \text { if } 0 \leq i \leq k-2 ; \\ (k, k-1,0,1, k), & \text { if } i=k-1 ; \\ (k, k, 1,2, k-1), & \text { if } i=k ; \\ (2 k-i, 2 k-i+1, i-k+1, & \text { if } k+1 \leq i \leq 2 k-2 ; \\ i-k+2,2 k-i-1), & \text { if } i=2 k-1 ;\end{cases}
$$

$r\left(v_{n} v_{3} \mid W_{E}\right)=(1,1, k, k-1,2)$,

$$
r\left(v_{3 i} v_{3 i+3} \mid W_{E}\right)= \begin{cases}(i+1, i, k-i, k-i-1, i+2), & \text { if } 1 \leq i \leq k-2 ; \\ (k, k-1,1,0, k), & \text { if } i=k-1 ; \\ (k, k, 1,0, k-1), & \text { if } i=k ; \\ (2 k-i, 2 k-i+1, i-k+1, i-k, & \\ 2 k-i-1), & \text { if } k+1 \leq i \leq 2 k-1 ;\end{cases}
$$

and $r\left(v_{n-2} v_{1} \mid W_{E}\right)=(0,1, k, k, 0)$. 
We see that there are no two edges having the same representations. This shows that $\operatorname{edim}\left(C_{n}(1,3)\right) \leq 5$.

On the other hand, we have to show that $\operatorname{edim}\left(C_{n}(1,3)\right) \geq 5$. For this purpose, we have to show that there is no edge metric generator have cardinality 4 , we suppose on contrary that $\operatorname{edim}\left(C_{n}(1,3)\right)=4$ and let $W_{E}=\left\{v_{1}, v_{i}, v_{j}, v_{l}\right\}$. Then the Table 7 shows all order pairs of edges $(e, f)$ for which $r\left(e \mid W_{E}\right)=r\left(f \mid W_{E}\right)$.

\begin{tabular}{|c|c|}
\hline Conditions on $i, j$ and $l$ & $(e, f)$ \\
\hline $2 \leq i \leq j \leq l \leq \frac{n}{2}$ and $j, l \neq \frac{n}{2}-1$ & $\left(v_{n} v_{1}, v_{n-2} v_{1}\right)$ \\
\hline $2 \leq i \leq j \leq l \leq \frac{n}{2}$ and $j, l \neq \frac{n}{2}-2$ & $\left(v_{n-1} v_{n}, v_{n-3} v_{n}\right)$ \\
\hline $2 \leq i \leq j \leq l \leq \frac{n}{2}$ and $i \neq 2$ & $\left(v_{\frac{n}{2}} v_{\frac{n}{2}+1}, v_{\frac{n}{2}} v_{\frac{n}{2}+3}\right)$ \\
\hline $2 \leq i \leq j \leq l \leq \frac{n}{2}$ and $i \neq 3$ & $\left(v_{\frac{n}{2}+1} v_{\frac{n}{2}+2}, v_{\frac{n}{2}+1} v_{\frac{n}{2}+4}\right)$ \\
\hline $\begin{array}{c}2 \leq i \leq j \leq \frac{n}{2}, \frac{n}{2}+1 \leq l \leq n \text { and } j \neq \frac{n}{2}-1, \text { also } \\
l \neq\left\{\frac{n}{2}+2, \frac{n}{2}+5, \frac{n}{2}+8, \ldots, n-2, n\right\}\end{array}$ & $\left(v_{n} v_{1}, v_{n-2} v_{1}\right)$ \\
\hline $\begin{array}{c}2 \leq i \leq j \leq \frac{n}{2}, \frac{n}{2}+1 \leq l \leq n \text { and } j \neq \frac{n}{2}-2, \text { also } \\
\quad l \neq\left\{\frac{n}{2}+1, \frac{n}{2}+4, \frac{n}{2}+7, \ldots, n-3, n-1\right\}\end{array}$ & $\left(v_{n-1} v_{n}, v_{n-3} v_{n}\right)$ \\
\hline $\begin{array}{c}2 \leq i \leq j \leq \frac{n}{2}, \frac{n}{2}+1 \leq l \leq n \text { and } i \neq 2, \text { also } \\
l \neq\left\{\frac{n}{2}+1, \frac{n}{2}+3, \frac{n}{2}+6, \frac{n}{2}+9, \ldots, n-1\right\}\end{array}$ & $\left(v_{\frac{n}{2}} v_{\frac{n}{2}+1}, v_{\frac{n}{2}} v_{\frac{n}{2}+3}\right)$ \\
\hline $\begin{array}{c}2 \leq i \leq j \leq \frac{n}{2}, \frac{n}{2}+1 \leq l \leq n \text { and } i \neq 3, \text { also } \\
l \neq\left\{\frac{n}{2}+2, \frac{n}{2}+4, \frac{n}{2}+7, \frac{n}{2}+10, \ldots, n\right\}\end{array}$ & $\left(v_{\frac{n}{2}+1} v_{\frac{n}{2}+2}, v_{\frac{n}{2}+1} v_{\frac{n}{2}+4}\right)$ \\
\hline
\end{tabular}

Table 7: $(e, f)$ for which $r\left(e \mid W_{E}\right)=r\left(f \mid W_{E}\right)$

In all possibilities, we get contradiction so it concludes that there is no edge metric generator of 4 vertices. Hence $\operatorname{edim}\left(C_{n}(1,3)\right)=5$, for $n \equiv 2(\bmod 6)$.

Case (vi): When $n \equiv 3(\bmod 6)$ and $n \geq 9$.

Let $n=6 k+3, k \geq 1, k \in \mathbf{Z}^{+}$and $W_{E}=\left\{v_{1}, v_{2}, v_{3}, v_{4}\right\} \subset V\left(C_{n}(1,3)\right)$, we have to show that $W_{E}$ is an edge metric generator of $C_{n}(1,3)$. For this, following are the representations of each edge of $C_{n}(1,3)$.

$$
r\left(v_{3 i+1} v_{3 i+2} \mid W_{E}\right)= \begin{cases}(0,0,1,1), & \text { if } i=0 ; \\ (i, i, i, i-1), & \text { if } 1 \leq i \leq k ; \\ (k, k, k+1, k), & \text { if } i=k+1 ; \\ (2 k-i+1,2 k-i+1,2 k-i+2, & \\ 2 k-i+2), & \text { if } k+2 \leq i \leq 2 k ;\end{cases}
$$


$r\left(v_{3 i+2} v_{3 i+3} \mid W_{E}\right)= \begin{cases}(1,0,0,1), & \text { if } i=0 ; \\ (i+1, i, i, i), & \text { if } 1 \leq i \leq k ; \\ 2 k-i+2), & \end{cases}$
$r\left(v_{3 i} v_{3 i+1} \mid W_{E}\right)= \begin{cases}(i, i, i-1, i-1), & \text { if } k+1 \leq i \leq 2 k ; \\ (k, k+1, k, k), & \text { if } 1 \leq i \leq k ; \\ (2 k-i+1,2 k-i+2,2 k-i+2, & \text { if } i=k+1 ; \\ 2 k-i+2), & \text { if } k+2 \leq i \leq 2 k ;\end{cases}$ $r\left(v_{n} v_{1} \mid W_{E}\right)=(0,1,1,1)$,

$r\left(v_{3 i+1} v_{3 i+4} \mid W_{E}\right)= \begin{cases}(0,1,1,0), & \text { if } i=0 ; \\ (i, i+1, i, i-1), & \text { if } 1 \leq i \leq k ; \\ (2 k-i, 2 k-i+1,2 k-i+2, & \\ 2 k-i+1), & \text { if } k+1 \leq i \leq 2 k-1 ;\end{cases}$

$r\left(v_{n-2} v_{1} \mid W_{E}\right)=(0,1,2,1)$

$r\left(v_{3 i+2} v_{3 i+5} \mid W_{E}\right)= \begin{cases}(1,0,1,1), & \text { if } i=0 ; \\ (i+1, i, i+1, i), & \text { if } 1 \leq i \leq k ; \\ (2 k-i+1,2 k-i, 2 k-i+1, & \\ 2 k-i+2), & \text { if } k+1 \leq i \leq 2 k ;\end{cases}$

$r\left(v_{n-1} v_{2} \mid W_{E}\right)=(1,0,1,2)$,

$r\left(v_{3 i} v_{3 i+3} \mid W_{E}\right)= \begin{cases}(i+1, i, i-1, i), & \text { if } 1 \leq i \leq k ; \\ (2 k-i+1,2 k-i+2,2 k-i+1, & \\ 2 k-i+2), & \text { if } k+1 \leq i \leq 2 k ;\end{cases}$

and $r\left(v_{n} v_{3} \mid W_{E}\right)=(1,1,0,1)$.

We see that there are no two edges having the same representations. This shows that $\operatorname{edim}\left(C_{n}(1,3)\right) \leq 4$.

On the other hand, we have to show that $\operatorname{edim}\left(C_{n}(1,3)\right) \geq 4$. For this purpose, we have to show that there is no edge metric generator have cardinality 3 , we suppose on contrary that edim $\left(C_{n}(1,3)\right)=3$ and let $W_{E}=\left\{v_{1}, v_{i}, v_{j}\right\}$. Then the Table 8 shows all order pairs of edges $(e, f)$ for which $r\left(e \mid W_{E}\right)=r\left(f \mid W_{E}\right)$. 


\begin{tabular}{|c|c|}
\hline Conditions on $i$ and $j$ & $(e, f)$ \\
\hline $2 \leq i \leq j \leq \frac{n+1}{2}$ & $\left(v_{n} v_{1}, v_{n-2} v_{1}\right)$ \\
\hline $2 \leq i \leq \frac{n-1}{2}, \frac{n+1}{2} \leq j \leq n$ and & \\
$j \neq\left\{\frac{n+1}{2}+2, \frac{n+1}{2}+5, \frac{n+1}{2}+8, \ldots, n-2, n\right\}$ & $\left(v_{n} v_{1}, v_{n-2} v_{1}\right)$ \\
\hline $2 \leq i \leq \frac{n-1}{2}, \frac{n+1}{2} \leq j \leq n$ and & \\
$j \neq\left\{\frac{n+1}{2}+1, \frac{n+1}{2}+4, \frac{n+1}{2}+7, \ldots, n-3, n-1\right\}$ & $\left(v_{n-1} v_{n}, v_{n-3} v_{n}\right)$ \\
\hline
\end{tabular}

Table 8: $(e, f)$ for which $r\left(e \mid W_{E}\right)=r\left(f \mid W_{E}\right)$

In all possibilities, we get contradiction so it concludes that there is no edge metric generator of 3 vertices. Hence $\operatorname{edim}\left(C_{n}(1,3)\right)=4$, for $n \equiv 3(\bmod 6)$. Case (vii): When $n \equiv 4(\bmod 6)$ and $n \geq 10$.

Let $n=6 k+4, k \geq 1, k \in \mathbf{Z}^{+}$and $W_{E}=\left\{v_{1}, v_{2}, v_{3}, v_{4}\right\} \subset V\left(C_{n}(1,3)\right)$, we have to show that $W_{E}$ is an edge metric generator of $C_{n}(1,3)$. For this, following are the representations of each edge of $C_{n}(1,3)$.

$$
\begin{aligned}
& r\left(v_{3 i+1} v_{3 i+2} \mid W_{E}\right)= \begin{cases}(0,0,1,1), & \text { if } i=0 ; \\
(i, i, i, i-1), & \text { if } 1 \leq i \leq k ; \\
(k, k+1, k+1, k), & \text { if } i=k+1 ; \\
(2 k-i+1,2 k-i+2,2 k-i+2, & \\
2 k-i+2), & \text { if } k+2 \leq i \leq 2 k ;\end{cases} \\
& r\left(v_{n} v_{1} \mid W_{E}\right)=(0,1,1,1),
\end{aligned}
$$$$
r\left(v_{3 i+2} v_{3 i+3} \mid W_{E}\right)= \begin{cases}(1,0,0,1), & \text { if } i=0 ; \\ (i+1, i, i, i), & \text { if } 1 \leq i \leq k ; \\ (2 k-i+1,2 k-i+1,2 k-i+2, & \\ 2 k-i+2), & \text { if } k+1 \leq i \leq 2 k ;\end{cases}
$$$$
r\left(v_{3 i} v_{3 i+1} \mid W_{E}\right)= \begin{cases}(i, i, i-1, i-1), & \text { if } 1 \leq i \leq k ; \\ (2 k-i+2,2 k-i+2,2 k-i+2, & \\ 2 k-i+3), & \text { if } k+2 \leq i \leq 2 k\end{cases}
$$$$
r\left(v_{3 i+1} v_{3 i+4} \mid W_{E}\right)= \begin{cases}(0,1,1,0), & \text { if } i=0 ; \\ (i, i+1, i, i-1), & \text { if } 1 \leq i \leq k ; \\ (k, k+1, k, k), & \text { if } i=k+1 ; \\ (2 k-i+1,2 k-i+2,2 k-i+1, & \\ 2 k-i+2), & \text { if } k+2 \leq i \leq 2 k ;\end{cases}
$$

$r\left(v_{n} v_{3} \mid W_{E}\right)=(1,1,0,1)$, 


$$
\begin{aligned}
& r\left(v_{3 i+2} v_{3 i+5} \mid W_{E}\right)= \begin{cases}(1,0,1,1), & \text { if } i=0 ; \\
(i+1, i, i+1, i), & \text { if } 1 \leq i \leq k-1 ; \\
(k, k, k+1, k), & \text { if } i=k ; \\
(2 k-i, 2 k-i+1,2 k-i+2, & \\
2 k-i+1), & \text { if } k+1 \leq i \leq 2 k-1 ;\end{cases} \\
& r\left(v_{n-2} v_{1} \mid W_{E}\right)=(0,1,2,1), \\
& r\left(v_{3 i} v_{3 i+3} \mid W_{E}\right)= \begin{cases}(i+1, i, i-1, i), & \text { if } 1 \leq i \leq k ; \\
(k+1, k, k, k+1), & \text { if } i=k+1 ; \\
(2 k-i+2,2 k-i+1,2 k-i+2, & \\
2 k-i+3), & \text { if } k+2 \leq i \leq 2 k ;\end{cases}
\end{aligned}
$$

and $r\left(v_{n-1} v_{2} \mid W_{E}\right)=(1,0,1,2)$.

We see that there are no two edges having the same representations. This shows that $\operatorname{edim}\left(C_{n}(1,3)\right) \leq 4$.

On the other hand, we have to show that $\operatorname{edim}\left(C_{n}(1,3)\right) \geq 4$. For this purpose, we have to show that there is no edge metric generator have cardinality 3 , we suppose on contrary that $\operatorname{edim}\left(C_{n}(1,3)\right)=3$ and let $W_{E}=\left\{v_{1}, v_{i}, v_{j}\right\}$. Then the Table 9 shows all order pairs of edges $(e, f)$ for which $r\left(e \mid W_{E}\right)=r\left(f \mid W_{E}\right)$.

\begin{tabular}{|c|c|}
\hline Conditions on $i$ and $j$ & $(e, f)$ \\
\hline $2 \leq i \leq j \leq \frac{n}{2}-1$ & $\left(v_{n} v_{1}, v_{n-2} v_{1}\right)$ \\
\hline $2 \leq i \leq j \leq \frac{n}{2}$ and $j \neq \frac{n}{2}-1$ & $\left(v_{n-1} v_{n}, v_{n-3} v_{n}\right)$ \\
\hline $2 \leq i \leq \frac{n}{2}-1, \frac{n}{2}+1 \leq j \leq n$ and & \\
$j \neq\left\{\frac{n}{2}+3, \frac{n}{2}+6, \frac{n}{2}+9, \ldots, n-2, n\right\}$ & $\left(v_{n} v_{1}, v_{n-2} v_{1}\right)$ \\
\hline $2 \leq i \leq \frac{n}{2}, \frac{n}{2}+1 \leq j \leq n$ and $i \neq \frac{n}{2}-1$, also \\
$j \neq\left\{\frac{n}{2}+2, \frac{n}{2}+5, \frac{n}{2}+8, \ldots, n-3, n-1\right\}$ & $\left(v_{n-1} v_{n}, v_{n-3} v_{n}\right)$ \\
\hline$i=\frac{n}{2}, \frac{n}{2}+1 \leq j \leq n$ and $j \neq \frac{n}{2}+2$ & $\left(v_{1} v_{2}, v_{1} v_{4}\right)$ \\
\hline$i=v_{\frac{n}{2}}$ and $j=v_{\frac{n}{2}+2}$ & $\left(v_{\frac{n}{2}+2} v_{\frac{n}{2}+3}, v_{\frac{n}{2}+2} v_{\frac{n}{2}+5}\right)$ \\
\hline$i=v_{\frac{n}{2}-1}, \frac{n}{2}+1 \leq j \leq n$ and $j \neq \frac{n}{2}+3$ & $\left(v_{2} v_{3}, v_{2} v_{5}\right)$ \\
\hline$i=v_{\frac{n}{2}}-1$ and $j=v_{\frac{n}{2}+3}$ & $\left(v_{\frac{n}{2}} v_{\frac{n}{2}}+1, v_{\frac{n}{2}}+1 v_{\frac{n}{2}}+2\right)$ \\
\hline
\end{tabular}

Table 9: $(e, f)$ for which $r\left(e \mid W_{E}\right)=r\left(f \mid W_{E}\right)$

In all possibilities, we get contradiction so it concludes that there is no edge metric generator of 3 vertices. Hence $\operatorname{edim}\left(C_{n}(1,3)\right)=4$, for $n \equiv 4(\bmod 6)$. Case (viii): When $n \equiv 5(\bmod 6)$ and $n \geq 11$.

Let $n=6 k+5, k \geq 1, k \in \mathbf{Z}^{+}$and $W_{E}=\left\{v_{1}, v_{2}, v_{\frac{n-1}{2}-1}, v_{\frac{n-1}{2}}, v_{n}\right\} \subset$ $V\left(C_{n}(1,3)\right)$, we have to show that $W_{E}$ is an edge metric generator of $C_{n}(1,3)$. 
For this, we give representations of each edge of $C_{n}(1,3)$.

Representation for edges is

$r\left(v_{3 i+1} v_{3 i+2} \mid W_{E}\right)= \begin{cases}(i, i, k-i, k-i, i+1), & \text { if } 0 \leq i \leq k ; \\ (2 k-i+2,2 k-i+2, i-k, & \text { if } k+1 \leq i \leq 2 k+1 ;\end{cases}$
$r\left(v_{3 i+2} v_{3 i+3} \mid W_{E}\right)= \begin{cases}(i+1, i, k-i, k-i, i+1), & \text { if } 0 \leq i \leq k-1 ; \\ (k+1, k, 1,0, k+1), & \text { if } i=k ; \\ (2 k-i+1,2 k-i+2, i-k+1, & \text { if } k+1 \leq i \leq 2 k ;\end{cases}$

$r\left(v_{n} v_{1} \mid W_{E}\right)=(0,1, k, k+1,0)$,

$r\left(v_{3 i} v_{3 i+1} \mid W_{E}\right)= \begin{cases}(i, i, k-i, k-i+1, i), & \text { if } 1 \leq i \leq k ; \\ (k+1, k+1,1,1, k+1), & \text { if } i=k+1 ; \\ (2 k-i+2,2 k-i+2, i-k, & \\ i-k, 2 k-i+2), & \text { if } k+2 \leq i \leq 2 k+1 ;\end{cases}$

$r\left(v_{3 i+1} v_{3 i+4} \mid W_{E}\right)= \begin{cases}(i, i+1, k-i-1, k-i, i+1), & \text { if } 0 \leq i \leq k-1 ; \\ (k, k+1,0,1, k+1), & \text { if } i=k ; \\ (2 k-i+2,2 k-i+1, i-k, & \\ i-k+1,2 k-i+1), & \text { if } k+1 \leq i \leq 2 k ;\end{cases}$

$r\left(v_{n-1} v_{2} \mid W_{E}\right)=(1,0, k+1, k, 1)$,

$r\left(v_{3 i+2} v_{3 i+5} \mid W_{E}\right)= \begin{cases}(i+1, i, k-i, k-i-1, i+2), & \text { if } 0 \leq i \leq k-1 ; \\ (k+1, k, 1,0, k), & \text { if } i=k ; \\ (2 k-i+1,2 k-i+2, i-k+1, & \\ i-k, 2 k-i), & \text { if } k+1 \leq i \leq 2 k ;\end{cases}$

$r\left(v_{n} v_{3} \mid W_{E}\right)=(1,1, k, k+1,0)$,

$r\left(v_{3 i} v_{3 i+3} \mid W_{E}\right)= \begin{cases}(i+1, i, k-i, k-i+1, i), & \text { if } 1 \leq i \leq k ; \\ (k+1, k, 1,1, k), & \text { if } i=k ; \\ (2 k-i+1,2 k-i+2, i-k+1, & \\ i-k, 2 k-i+2), & \text { if } k+1 \leq i \leq 2 k ;\end{cases}$

and $r\left(v_{n-2} v_{1} \mid W_{E}\right)=(0,1, k, k+1,1)$.

We see that there are no two edges having the same representations. This shows that $\operatorname{edim}\left(C_{n}(1,3)\right) \leq 5$.

On the other hand, we have to show that $\operatorname{edim}\left(C_{n}(1,3)\right) \geq 5$. For this purpose, we have to show that there is no edge metric generator have cardinality 4 , we 
suppose on contrary that $\operatorname{edim}\left(C_{n}(1,3)\right)=4$ and let $W_{E}=\left\{v_{1}, v_{i}, v_{j}, v_{l}\right\}$. Then the Table 10 shows all order pairs of edges $(e, f)$ for which $r\left(e \mid W_{E}\right)=$ $r\left(f \mid W_{E}\right)$.

\begin{tabular}{|c|c|}
\hline Conditions on $i, j$ and $l$ & $(e, f)$ \\
\hline $2 \leq i \leq j \leq l \leq \frac{n}{2}$ and $j, l \neq \frac{n}{2}-1$ & $\left(v_{n} v_{1}, v_{n-2} v_{1}\right)$ \\
\hline $2 \leq i \leq j \leq l \leq \frac{n+1}{2}$, and $j, l \neq \frac{n-1}{2}$ & $\left(v_{n-1} v_{n}, v_{n-3} v_{n}\right)$ \\
\hline$j=v_{\frac{n-1}{2}}, l=v_{\frac{n+1}{2}}, 2 \leq i \leq \frac{n-3}{2}$ and $i \neq 2$ & $\left(v_{\frac{n+3}{2}} v_{\frac{n+5}{2}}, v_{\frac{n+3}{2}} v_{\frac{n+9}{2}}\right)$ \\
\hline$i=2, j=v_{\frac{n-1}{2}}$ and $l=v_{\frac{n+1}{2}}$ & $\left(v_{n-1} v_{n-2}, v_{n-1} v_{n-4}\right)$ \\
\hline $2 \leq i \leq j \leq \frac{n-1}{2}, \frac{n+1}{2} \leq l \leq n$ and & \\
\hline$l \neq\left\{\frac{n+1}{2}, \frac{n+1}{2}+3, \frac{n+1}{2}+6, \frac{n+1}{2}+9, \ldots, n-2, n\right\}$ & $\left(v_{n} v_{1}, v_{n-2} v_{1}\right)$ \\
\hline $2 \leq i \leq j \leq \frac{n-1}{2}-1, \frac{n+1}{2} \leq l \leq n$ and $j \neq \frac{n-1}{2}$, also & \\
$l \neq\left\{\frac{n+1}{2}+2, \frac{n+1}{2}+5, \frac{n+1}{2}+8, \frac{n+1}{2}+11, \ldots, n-3, n-1\right\}$ & $\left(v_{n-1} v_{n}, v_{n-3} v_{n}\right)$ \\
\hline$j=v_{\frac{n-1}{2}}, 2 \leq i \leq \frac{n-3}{2}, \frac{n+1}{2} \leq l \leq n$ and & \\
$l \neq \frac{n+3}{2}$, also $i \neq\left\{2,4,7,10, \ldots, \frac{n-3}{2}\right\}$ & $\left(v_{1} v_{2}, v_{1} v_{4}\right)$ \\
\hline$j=v_{\frac{n-1}{2}}, 2 \leq i \leq \frac{n-3}{2}, \frac{n+1}{2} \leq l \leq n$ and & $\left(v_{\frac{n-1}{2}-1} v_{\frac{n-1}{2}}\right.$, \\
$l \neq n-1$, also $i \neq\left\{2,5,8, \ldots, \frac{n-7}{2}, \frac{n-3}{2}\right\}$ & $\left.v_{\frac{n-1}{2}-3} v_{\frac{n-1}{2}}\right)$ \\
\hline$j=v_{\frac{n-1}{2}}, 2 \leq i \leq \frac{n-3}{2}, \frac{n+1}{2} \leq l \leq n$ and & $\left(v_{\frac{n-1}{2}} v_{\frac{n+1}{2}}\right.$, \\
$l \neq\left\{\frac{n+1}{2}, \frac{n+1}{2}+2, \frac{n+1}{2}+5, \frac{n+1}{2}+8, \ldots, n\right\}$ & $v_{\frac{n-1}{2}} v_{\frac{n-1}{2}}+3$ \\
\hline
\end{tabular}

Table 10: $(e, f)$ for which $r\left(e \mid W_{E}\right)=r\left(f \mid W_{E}\right)$

In all possibilities, we get contradiction so it concludes that there is no edge metric generator of 4 vertices. Hence $\operatorname{edim}\left(C_{n}(1,3)\right)=5$, for $n \equiv 5(\bmod 6)$.

\section{Conclusion}

In this paper, we have studied the edge metric dimension of circulant graphs $C_{n}(1,2)$ and $C_{n}(1,3)$. It is observed that the edge metric dimension of these graphs is constant and does not depend on the number of vertices.

Open Problem: Calculate the edge metric dimension of $C_{n}(1,2,3)$.

\section{Acknowledgement}

The authors are grateful to the reviewers for the careful reading to improve the manuscript. 


\section{References}

[1] J. Caceres, C. Hernando, M. Mora, I.M. Pelayo, M.L. Puertas, C. Seara, D.R. Wood. On the metric dimension of cartesian products of graphs. SIAM J. Discrete Math., 21(2): 423-441, 2007.

[2] G. Chartrand, C. Poisson, P. Zhang. Resolvability and the upper dimension of graphs. Comput. Math. with Appl., 39(12): 19-28, 2000.

[3] K. Chau, S. Gosselin. The metric dimension of circulant graphs and their cartesian products. Opuscula Mathematica, 37(4): 509-534, 2017.

[4] F. Haray, R. A. Melter. On the metric dimension of a graph. Ars Combin., 2(1): 191-195, 1976.

[5] S. Hoffmann, A. Elterman, E. Wanke. A linear time algorithm for metric dimension of cactus block graphs. Theor. Comput. Sci., 630: 43-62, 2016.

[6] I. Javaid, M. N. Azhar, M. Salman. Metric dimension and determining number of Cayley graphs. World Appl. Sci. J., 18: 1800-1812, 2012.

[7] I. Javaid, M. T. Rahim, K. Ali. Families of regular graphs with constant metric dimension. Utilitas Math., 75: 21-33, 2008.

[8] I. Javaid, N. K. Raja, M. Salman, M. N. Azhar. The partition dimension circulant graphs. World Appl. Sci. J., 18(12): 1705-1717, 2012.

[9] G. Jager, F. Drewes. The metric dimension of $Z_{n} \times Z_{n} \times Z_{n}$ is $\lfloor 3 n / 2\rfloor$. Theor. Comput. Sci., https://doi.org/10.1016/j.tcs.2019.05.042, 2019.

[10] M. Johnson. Structure-activity maps for visualizing the graph variables arising in drug design. Biopharmaceutical Stat., 3(2): 203-236, 1993.

[11] A. Kelenc, N. Tratnik, I. G. Yero. Uniquely identifying the edges of a graph: the edge metric dimension. Discrete Appl. Math., 251: 204-220, 2018 .

[12] S. Khuller, B. Raghavachari, A. Rosenfeld. Landmarks in graphs. Discrete Appl. Math., 70(3): 217-229, 1996.

[13] J. Kratica, V. Filipovi, A. Kartelj. Edge Metric Dimension of Some Generalized Petersen Graphs. http://arxiv.org/abs/1807.00580v1, 2018.

[14] R. A. Melter and I. Tomescu. Metric bases in digital geometry. Comput. Vision, Graphics, Image Process., 25(1): 113-121, 1984. 
[15] Z. Mufti, M. Nadeem, A. Ahmad, Z. Ahmad. Computation of edge metric dimension of barcycentric subdivision of Cayley graphs. ffhal$01902772 f, 2018$.

[16] M. Salman, I. Javid, M.A. Chaudhry. Resolvability in circulant graphs. Acta Math. Sin. Engl. Ser., 28: 1851-1864, 2012.

[17] A. Seb, E. Tannier. On metric generators of graphs. Math. Oper. Res., 29: 383-393, 2004.

[18] P. J. Slater. Leaves of trees. Congr. Numer., 14(37): 549-559, 1975.

[19] P. J. Slater. Dominating and reference sets in graphs. J. Math. Phys. Sci., 22: 445-455, 1988.

[20] S. Zejnilovic, D. Mitsche, J. Gomes, B. Sinopoli. Extending the metric dimension to graphs with missing edges. Theor. Comput. Sci., 609: 384$394,2016$.

[21] N. Zubrilina. On the edge dimension of a graph. Discrete Math., 341(7): 2083-2088, 2018.

Muhammad Ahsan,

Department of Mathematics,

University of Management and Technology (UMT),

Lahore Pakistan

Email: muhammadahsanm@gmail.com

Zohaib Zahid,

Department of Mathematics,

University of Management and Technology (UMT),

Lahore Pakistan

Email: zohaib_zahid@hotmail.com

Sohail Zafar,

Department of Mathematics,

University of Management and Technology (UMT),

Lahore Pakistan

Email: sohailahmad04@gmail.com 